\title{
A NEW SLOPE INDEX FOR SOLVING NXM FLOW SHOP SEQUENCING PROBLEMS WITH MINIMUM MAKESPAN
}

\begin{abstract}
Summary
A flow shop sequencing problem is one of the classical problems in the production scheduling. In a flow shop, a particular case of manufacturing process follows a fixed linear structure. The purpose of this paper is to find the minimum total processing time (makespan) of sequencing ' $\mathrm{n}$ ' jobs on ' $\mathrm{m}$ ' machines for a flow shop problem in a static workshop. The proposed approach is based on the slope of each job on its journey from the first to the last machine. This approach is compared with five well-known heuristics (Palmer, Gupta, CDS, Dannenbring, Hundal) and one more recent technique that is based on the harmonic triangle. The results obtained from this study for different sizes of ' $n$ ' $x$ ' $m$ ' flow shop sequencing problems ranging from $4 \times 4$ to $50 \times 20$ indicate that the proposed approach is efficient with an encouraging percentage of improvements compared with all other six heuristic techniques.
\end{abstract}

Key words: $\quad$ scheduling, flow shop, sequencing, makespan, Gantt chart

\section{Introduction}

Fundamentally, the flow shop scheduling consists of a fixed linear structure of a particular case of a job shop. The process involves establishing a step-by-step procedure to be adhered to strictly and all job operations are to be performed in the exact order. Such scheduling is important in designing the layout of a new and current industrial production facilities due to its ability to reduce and eliminate wasteful idle machine time during operations[1].

Sequencing is a key in determining or selecting a particular order in which a limited number of machines can complete different jobs in the shortest possible time and the least expensive manner. Flow shop problems require sequencing of ' $n$ ' jobs by ' $m$ ' machines to reduce the overall completion time, known as makespan, for all jobs. The savings in time and production costs drive the search for improved sequencing in workshop scheduling, with the goal being increased productivity. This paper proposes a new job sequencing technique for the static flow shop problem based on the slope equation of each job on its journey from the first to the last machine.

This paper is organized as follows: Section 2 provides a brief historical overview of six job sequencing techniques and their associated equations. Section 3 presents a proposed technique for reducing the makespan time. A numerical example of the proposed method is presented in Section 4. Section 5 compares the proposed technique to other six alternative techniques in a tabular form. Discussion and conclusions comprise Section 6. 


\section{Six efficient heuristic procedures}

Over the past several decades, issues relating to the flow shop scheduling and job sequencing have generated extraordinary interest in operations research with a nearly inexhaustible development of new methods and improvement of the old ones. Increased automation in nearly every industry and nearly every facet of each organization's operations in a highly competitive global environment demands maximum optimization of all available resources. The only constant resource in all scenarios is time. Thus, the demand for the most efficient and effective way to optimize a project from beginning to end by applying advanced scheduling and sequencing techniques is paramount importance.

Johnson [2] first studied the flow shop problem for ' $n$ ' jobs to be processed on two machines. Completion of the job in its entirety represented the total completion time as an objective function. Johnson's solution, known as Johnson's rule, states that job $i$ precedes job $j$ in an optimal sequence if and only if $\min \left\{t_{i 1}, t_{j 2}\right\} \leq \min \left\{t_{i 2}, t_{j 1}\right\}$, where $t$ is the time interval that one machine has to devote to the job in order to complete it. The potential complexity of the flow shop scheduling is evident when ' $\mathrm{m}$ ' is greater than 2 and the problem, in this case, becomes non-deterministic polynomial-time hard (NP-hard).

Palmer [3] provided methods for reaching lower bounds, which the makespans must exceed or equal. The Palmer heuristic calculates a slope index $S_{i}$ for each job and then schedules the jobs in descending order given by these indices [4]. Formally, the slope index is:

$$
S_{i}=\sum_{j=1}^{M}(M+2 j+1) t_{i j} \quad \text { for } i=1,2, \ldots, N
$$

where $t_{i j}$ is the processing time of job $\mathrm{i}$ on machine $\mathrm{j}$.

Campbell, Dudek, and Smith [5], who developed the CDS heuristic, followed Johnson's algorithm. This heuristic involves solving M-1 two-machine problems by dividing $M$ machines into a set of two groups of the flow shop problem, then the authors applied Johnson's two-machine rule to find M-1 schedules in which the best schedule is selected. The processing times $P_{i \mathrm{~g}}^{k}$ of the $i$-th job on the g-th machine group for the $k$-th reduced problem, $\mathrm{g}=1$ or 2 and $k=1, \ldots, \mathrm{M}-1$, are:

$$
P_{i 1}^{k}=\sum_{j}^{k} t_{i j} \quad \text { and } \quad P_{i 2}^{k}=\sum_{j}^{k} t_{i, m-j+1}
$$

Gupta [6] designed a heuristic to solve a flow shop problem with a given number of ' $n$ ' jobs on a given number of ' $\mathrm{m}$ ' machines where the workflow is unidirectional. Such a scenario requires the technological order of all jobs and all machines be identical. Since the numbering of machines is random, the machine numbers can reflect the end goal that the jobs are processed first on machine 1 , then passed on to machine $2,3 \ldots$. , all the way to machine ' $\mathrm{m}$ ' as the last machine in a consecutive manner [7]. Gupta assigned an index $f_{i}$ to each job $i$; then sorted the jobs in ascending order given by these indices. He generalized the index as follows:

$$
f_{i}=\frac{A}{\min _{1 \leq m \leq M-1}\left(t_{i m}+t_{i m+1}\right)},
$$

where $\mathrm{A}=\left\{\begin{array}{cr}1 & \text { if } t_{\text {im }}<t_{\text {im+1 }} \\ -1 & \text { Otherwise }\end{array}\right.$.

Dannenbring [8] introduced a Rapid Access (RA) heuristic method. Dannenbring attempted to utilize the Campbell, Dudek, and Smith (CDS) heuristic methods and Palmer's slope index. Dannenbring constructed an artificial two-machine problem with the processing times reflecting the same behaviors as Palmer's slope index, then applied Johnson's algorithm. The constructed processing times were: 


$$
P_{i 1}=\sum_{j=1}^{M}(M-j+1) t_{i j}, \quad P_{i 2}=\sum_{j=1}^{M}(j) t_{i j} \quad \text { for } \mathrm{i}=1,2, \ldots, N
$$

The RA heuristic method was proposed to solve problems as quickly as possible. It effectively resolves permutations of flow shop problems and the overall time of completion (makespan). It only uses Johnson's rule to solve idle time for two auxiliary machines [9].

Hundal and Rajgopal [10] extended Palmer's heuristic by computing two other sets of slope indices. Consequently, two more schedules are produced, and the best one is selected. The two sets of slope indices are:

$$
S_{i}=\sum_{j=1}^{M}(M-2) t_{i j} \quad \text { and } \quad S_{i}=\sum_{j=1}^{M}(M-2 j+2) t_{i j} \quad \text { for } \mathrm{i}=1,2, \ldots, N
$$

Dhanasakkaravarthi and Krishnamoorthy [11] used a harmonic triangle that is similar to Pascal's triangle involving binomial coefficients. The harmonic triangle for the $r_{t h}$ entry in the $n_{t h}$ row is given mathematically by:

$$
H(n, r)=\frac{(r-1) !}{n(n-1)(n-2) \ldots(n-r+1)}=\frac{(r-1) !(n-r) !}{n !}
$$

Dhanasakkaravarthi and Krishnamoorthy used the harmonic triangle form to solve permutation flow shop sequencing problems by reducing ' $n$ ' jobs, ' $m$ ' machines to ' $n$ ' jobs, ' 2 ' machines, then the optimum makespan can be determined using Johnson's rule.

\section{Illustrative example of the proposed flow shop sequencing technique}

To illustrate the proposed heuristic technique for the static flow shop, the following assumptions are made:

- There are ' $n$ ' number of jobs (J) and ' $m$ ' number of machines (M).

- The order of sequence of operations of ' $n$ ' jobs on all ' $m$ ' machines is the same.

- The setup time is not considered for calculating the total processing time (makespan).

In Table 1, the data from Ajay and Rajan [12] are used and an eight-job and threemachine problem is presented. Each cell in the table represents the processing time $t_{i j}$ of an operation job $i$ on machine $m$.

Table 1 A numerical example for 8-job and 3-machine problem

\begin{tabular}{lccc}
\hline JM & Machine 1 & Machine 2 & Machine 3 \\
\hline Job 1 & 6 & 5 & 1 \\
Job 2 & 3 & 9 & 5 \\
Job 3 & 8 & 1 & 6 \\
Job 4 & 4 & 6 & 3 \\
Job 5 & 9 & 8 & 2 \\
Job 6 & 3 & 2 & 4 \\
Job 7 & 5 & 9 & 4 \\
Job 8 & 2 & 8 & 9 \\
\hline
\end{tabular}

Step \#1: Due to the fluctuation of the processing time for job i from one machine to another, calculate the slope of the trend line $T_{i}$ of the job i starting its journey from the first machine to the last machine as follows:

$$
T_{i}=\frac{t_{i m}-t_{i 1}}{m-1}
$$


For example, the slope of the trend line $T_{i}$ of job 1 , which starts on machine 1 , which requires a processing time of 6 minutes and ends in machine 3 with a processing time of 1 minute, can be calculated as follows:

$$
T_{1}=\frac{1-6}{3-1}=-2.5
$$

Step \#2: Assign slope index $S_{i}$ of job i by dividing its trend $\mathrm{T}_{\mathrm{i}}$ by its total processing times in ' $\mathrm{m}$ ' machines multiplied by 100 to clear the differences among the jobs. The slope index $S_{i}$ can be calculated as follows:

$$
S_{i}=\left(\frac{T_{i}}{\sum_{j=1}^{m} t_{i j}}\right) * 100
$$

For example, the value of $T_{1}$ from the previous step is -2.5 , and then the slope index $S_{i}$ of job 1 can be calculated as follows:

$$
S_{i}=\left(\frac{-2.5}{6+5+1}\right) * 100=-20.83
$$

Step \#3: Rank the jobs in descending order by their indices and calculate the total processing time. The rationale of using descending order is to delay the execution of the jobs that have low processing times on the production line and this correspondingly reduces the total idle times of the machines.

Table 2 shows all results obtained for all jobs, while Figure 1 shows the trend lines of the eight jobs. After ranking the job sequence in descending order the final job sequence result is $8-2-6-7-4-3-5-1$, and the makespan is 51. Figure 2 shows the flow of each job on each machine.

Table 2 Results (slope of the trend lines and slope indices) of eight jobs

\begin{tabular}{ccccccccc}
\hline Jobs & J1 & J2 & J3 & J4 & J5 & J6 & J7 & J8 \\
\hline$T_{i}$ & -2.5 & 1 & -1 & -0.5 & -3.5 & 0.5 & -0.5 & 3.5 \\
$S_{i}$ & -20.83 & 5.88 & -6.67 & -3.85 & -18.42 & 5.56 & -2.78 & 18.42 \\
Rank & 8 & 2 & 6 & 5 & 7 & 3 & 4 & 1 \\
\hline
\end{tabular}

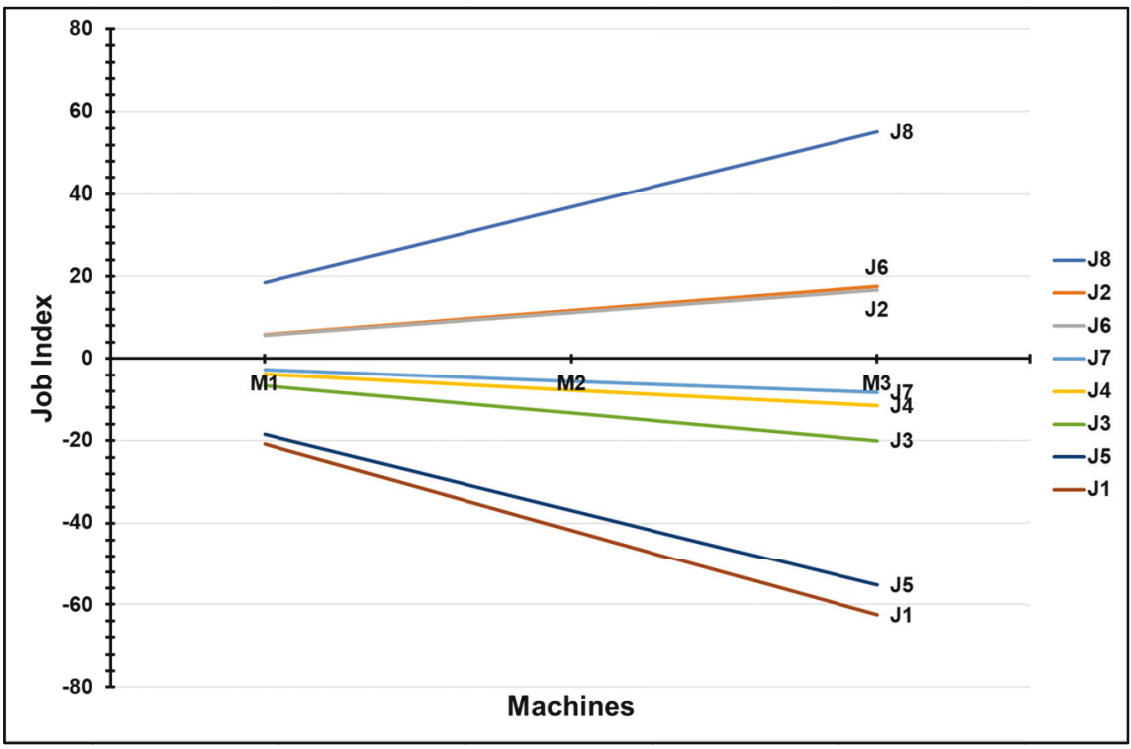

Fig. 1 Trend lines of the original problem of eight jobs and three machines 


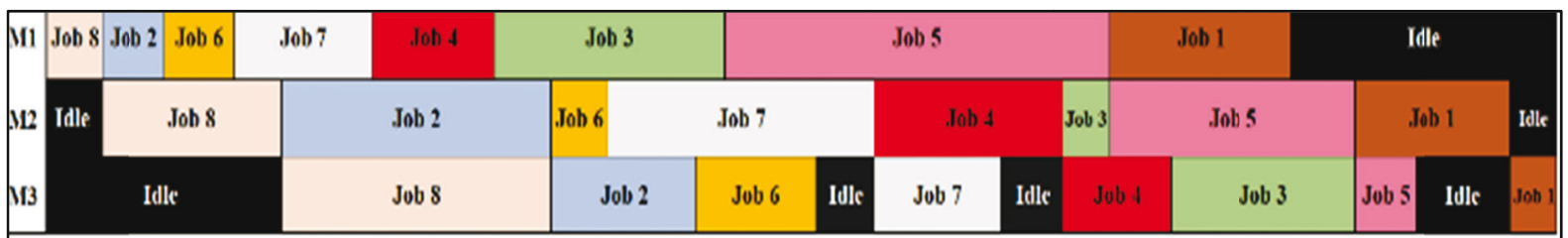

$123456789101112131415161718192021222324252627282930313233343536 \quad 3738394041424344454647484950 \quad 51$

Time

Fig. 2 Flow of each job on each machine

\section{Comparison of the proposed heuristic with six benchmark algorithms}

To study the effectiveness of the proposed heuristic technique, the six algorithms mentioned in Section 2 were compared with the proposed heuristic for minimum makespan. For this purpose, ten flow shop problems were selected from the literature and reproduced in Figure $3(\mathrm{a}-\mathrm{j})$. All ten problem sizes range from $4 \times 4$ to $50 \times 10$ to cover both small and large cases. The benchmarks of Taillard [13] and Vallade et al. [14] provided the parameters for the 20x10, 20x20, and 50x10 problems. The results (job sequence and corresponding makespan) obtained with the six benchmark methods are demonstrated in Figure 4.

\begin{tabular}{|c|c|c|c|c|}
\hline \multicolumn{5}{|c|}{ Case 1 (4x4): Dhanasakka ravarthi (2019) } \\
\hline J\M & M1 & M2 & M3 & M4 \\
\hline J1 & 4 & 3 & 7 & 8 \\
\hline J2 & 3 & 7 & 2 & 5 \\
\hline J3 & 1 & 2 & 4 & 7 \\
\hline J4 & 3 & 4 & 3 & 2 \\
\hline
\end{tabular}

(a)

\begin{tabular}{|c|c|c|c|c|c|c|}
\hline \multicolumn{7}{|c|}{ Case 3 (5x6): Mostafa Khatami (2019) } \\
\hline JM & $\mathrm{M} 1$ & $\mathrm{M} 2$ & $\mathrm{M} 3$ & $\mathrm{M} 4$ & $\mathrm{M} 5$ & $\mathrm{M} 6$ \\
\hline $\mathrm{J} 1$ & 2 & 3 & 15 & 3 & 8 & 8 \\
\hline $\mathrm{J} 2$ & 3 & 4 & 21 & 7 & 13 & 15 \\
\hline $\mathrm{J} 3$ & 9 & 12 & 30 & 15 & 19 & 21 \\
\hline $\mathrm{J} 4$ & 13 & 14 & 34 & 19 & 24 & 26 \\
\hline $\mathrm{J} 5$ & 15 & 16 & 37 & 24 & 28 & 35 \\
\hline
\end{tabular}

(c)

\begin{tabular}{|c|c|c|c|c|c|}
\hline \multicolumn{7}{|c|}{ Case 2 (5x5):Yunior (2017) } \\
\hline JM & M1 & M2 & M3 & M4 & M5 \\
\hline J1 & 10 & 11 & 6 & 8 & 11 \\
\hline J2 & 15 & 9 & 14 & 10 & 14 \\
\hline J3 & 12 & 11 & 9 & 10 & 6 \\
\hline J4 & 8 & 4 & 8 & 9 & 12 \\
\hline J5 & 6 & 6 & 8 & 6 & 3 \\
\hline
\end{tabular}

(b)

\begin{tabular}{|c|c|c|c|c|c|c|c|}
\hline \multicolumn{10}{|c|}{ Case 4 (7x7): Vladimír (2010) } \\
\hline J\M & M1 & M2 & M3 & M4 & M5 & M6 & M7 \\
\hline $\mathrm{J} 1$ & 3 & 2 & 4 & 5 & 1 & 3 & 5 \\
\hline $\mathrm{J} 2$ & 5 & 5 & 8 & 7 & 2 & 5 & 2 \\
\hline $\mathrm{J} 3$ & 7 & 8 & 1 & 6 & 8 & 4 & 8 \\
\hline $\mathrm{J} 4$ & 1 & 1 & 6 & 1 & 4 & 6 & 4 \\
\hline $\mathrm{J} 5$ & 6 & 6 & 7 & 8 & 6 & 8 & 6 \\
\hline $\mathrm{J} 6$ & 9 & 7 & 9 & 4 & 7 & 1 & 3 \\
\hline $\mathrm{J} 7$ & 4 & 9 & 1 & 3 & 4 & 2 & 2 \\
\hline
\end{tabular}

(d)

\begin{tabular}{|c|c|c|c|}
\hline \multicolumn{4}{|c|}{ Case 5 (8x3): Illustrative Example } \\
\hline $\mathrm{J} \backslash \mathrm{M}$ & $\mathrm{M} 1$ & $\mathrm{M} 2$ & $\mathrm{M} 3$ \\
\hline $\mathrm{J} 1$ & 6 & 5 & 1 \\
\hline $\mathrm{J} 2$ & 3 & 9 & 5 \\
\hline $\mathrm{J} 3$ & 8 & 1 & 6 \\
\hline $\mathrm{J} 4$ & 4 & 6 & 3 \\
\hline $\mathrm{J} 5$ & 9 & 8 & 2 \\
\hline $\mathrm{J} 6$ & 3 & 2 & 4 \\
\hline $\mathrm{J} 7$ & 5 & 9 & 4 \\
\hline $\mathrm{J} 8$ & 2 & 8 & 9 \\
\hline
\end{tabular}

(e)

\begin{tabular}{|c|c|c|c|c|c|c|c|c|}
\hline \multicolumn{1}{|c|}{ Case 6 (10x8): Ajay Kumar Agarwal (2013) } \\
\hline JM & M1 & M2 & M3 & M4 & M5 & M6 & M7 & M8 \\
\hline J1 & 6 & 5 & 1 & 7 & 9 & 3 & 4 & 2 \\
\hline J2 & 3 & 9 & 5 & 7 & 2 & 5 & 6 & 1 \\
\hline J3 & 8 & 1 & 6 & 4 & 3 & 9 & 5 & 9 \\
\hline J4 & 4 & 6 & 3 & 1 & 5 & 6 & 7 & 7 \\
\hline J5 & 9 & 8 & 2 & 9 & 2 & 5 & 9 & 6 \\
\hline J6 & 3 & 2 & 4 & 3 & 7 & 2 & 3 & 5 \\
\hline J7 & 5 & 9 & 4 & 2 & 4 & 8 & 6 & 6 \\
\hline J8 & 2 & 8 & 9 & 1 & 6 & 3 & 4 & 8 \\
\hline J9 & 1 & 4 & 6 & 2 & 5 & 4 & 3 & 9 \\
\hline J10 & 6 & 3 & 5 & 5 & 2 & 7 & 1 & 9 \\
\hline
\end{tabular}

(f) 


\begin{tabular}{|c|c|c|c|c|c|c|c|c|c|c|}
\hline \multicolumn{10}{|c|}{ Case 7 (10x10): Dhanasakkaravarthi (2019) } \\
\hline JM & $\mathrm{M} 1$ & $\mathrm{M} 2$ & $\mathrm{M} 3$ & $\mathrm{M} 4$ & $\mathrm{M} 5$ & $\mathrm{M} 6$ & $\mathrm{M} 7$ & $\mathrm{M} 8$ & $\mathrm{M} 9$ & $\mathrm{M} 10$ \\
\hline $\mathrm{J} 1$ & 5 & 2 & 3 & 5 & 7 & 9 & 7 & 8 & 2 & 7 \\
\hline $\mathrm{J} 2$ & 2 & 6 & 4 & 2 & 6 & 2 & 5 & 2 & 6 & 1 \\
\hline $\mathrm{J} 3$ & 1 & 2 & 2 & 1 & 3 & 7 & 2 & 5 & 4 & 4 \\
\hline $\mathrm{J} 4$ & 7 & 5 & 6 & 3 & 2 & 3 & 2 & 4 & 2 & 2 \\
\hline $\mathrm{J} 5$ & 6 & 6 & 1 & 8 & 6 & 4 & 3 & 9 & 6 & 4 \\
\hline $\mathrm{J} 6$ & 3 & 7 & 5 & 2 & 2 & 1 & 5 & 3 & 2 & 6 \\
\hline $\mathrm{J} 7$ & 7 & 2 & 4 & 6 & 5 & 5 & 1 & 2 & 5 & 2 \\
\hline J8 & 5 & 1 & 7 & 1 & 7 & 3 & 6 & 6 & 2 & 2 \\
\hline J9 & 7 & 8 & 6 & 9 & 1 & 8 & 2 & 1 & 6 & 6 \\
\hline $\mathrm{J} 10$ & 4 & 3 & 5 & 8 & 3 & 1 & 3 & 8 & 3 & 7 \\
\hline
\end{tabular}

(g)

\begin{tabular}{|c|c|c|c|c|c|c|c|c|c|c|}
\hline \multicolumn{10}{|c|}{ Case 8 (20x10): Taillard (1993) } \\
\hline JM & M1 & M2 & M3 & M4 & M5 & M6 & M7 & M8 & M9 & M10 \\
\hline J1 & 74 & 28 & 89 & 60 & 54 & 92 & 9 & 4 & 25 & 15 \\
\hline J2 & 21 & 3 & 52 & 88 & 66 & 11 & 8 & 18 & 15 & 84 \\
\hline J3 & 58 & 27 & 56 & 26 & 12 & 54 & 88 & 25 & 91 & 8 \\
\hline J4 & 4 & 61 & 13 & 58 & 57 & 97 & 72 & 28 & 49 & 30 \\
\hline J5 & 21 & 34 & 7 & 76 & 70 & 57 & 27 & 95 & 56 & 95 \\
\hline J6 & 28 & 76 & 32 & 98 & 82 & 53 & 22 & 51 & 10 & 79 \\
\hline J7 & 58 & 64 & 32 & 29 & 99 & 65 & 50 & 84 & 62 & 9 \\
\hline J8 & 83 & 87 & 98 & 47 & 84 & 77 & 2 & 18 & 70 & 91 \\
\hline J9 & 31 & 54 & 46 & 79 & 16 & 51 & 49 & 6 & 76 & 76 \\
\hline J10 & 61 & 98 & 60 & 26 & 41 & 36 & 82 & 90 & 99 & 26 \\
\hline J11 & 94 & 76 & 23 & 19 & 23 & 53 & 93 & 69 & 58 & 42 \\
\hline J12 & 44 & 41 & 87 & 48 & 11 & 19 & 96 & 61 & 83 & 66 \\
\hline J13 & 97 & 70 & 7 & 95 & 68 & 54 & 43 & 57 & 84 & 70 \\
\hline J14 & 94 & 43 & 36 & 78 & 58 & 86 & 13 & 5 & 64 & 91 \\
\hline J15 & 66 & 42 & 26 & 77 & 30 & 40 & 60 & 75 & 74 & 67 \\
\hline J16 & 6 & 79 & 85 & 90 & 5 & 56 & 11 & 4 & 14 & 3 \\
\hline J17 & 37 & 88 & 7 & 24 & 5 & 79 & 37 & 38 & 18 & 98 \\
\hline J18 & 22 & 15 & 34 & 10 & 39 & 74 & 91 & 28 & 48 & 4 \\
\hline J19 & 99 & 49 & 36 & 85 & 58 & 24 & 84 & 4 & 96 & 71 \\
\hline J20 & 83 & 72 & 48 & 55 & 31 & 3 & 67 & 80 & 86 & 62 \\
\hline
\end{tabular}

(h)

\begin{tabular}{|c|c|c|c|c|c|c|c|c|c|c|c|c|c|c|c|c|c|c|c|c|}
\hline \multicolumn{10}{|c|}{ Case 9 (20x20): Taillard (1993) } \\
\hline JMM & M1 & M2 & M3 & M4 & M5 & M6 & M7 & M8 & M9 & M10 & M11 & M12 & M13 & M14 & M15 & M16 & M17 & M18 & M19 & M20 \\
\hline J1 & 50 & 90 & 39 & 34 & 66 & 81 & 27 & 48 & 46 & 68 & 48 & 92 & 78 & 84 & 93 & 39 & 43 & 1 & 65 & 87 \\
\hline J2 & 78 & 56 & 9 & 43 & 84 & 73 & 66 & 38 & 83 & 57 & 97 & 52 & 77 & 13 & 12 & 2 & 65 & 93 & 39 & 1 \\
\hline J3 & 36 & 43 & 10 & 19 & 55 & 48 & 85 & 70 & 82 & 39 & 91 & 82 & 85 & 17 & 6 & 54 & 87 & 85 & 4 & 72 \\
\hline J4 & 85 & 88 & 60 & 98 & 4 & 99 & 53 & 21 & 33 & 53 & 63 & 18 & 45 & 29 & 43 & 41 & 80 & 4 & 31 & 19 \\
\hline J5 & 9 & 92 & 98 & 44 & 51 & 8 & 31 & 15 & 47 & 31 & 80 & 83 & 20 & 84 & 69 & 49 & 93 & 39 & 13 & 88 \\
\hline J6 & 75 & 64 & 96 & 95 & 22 & 41 & 26 & 33 & 68 & 9 & 81 & 28 & 61 & 69 & 37 & 57 & 36 & 80 & 96 & 74 \\
\hline J7 & 46 & 94 & 6 & 19 & 20 & 51 & 85 & 92 & 43 & 75 & 70 & 70 & 36 & 31 & 76 & 63 & 89 & 46 & 25 & 88 \\
\hline J8 & 73 & 3 & 56 & 73 & 80 & 82 & 36 & 98 & 90 & 46 & 10 & 46 & 65 & 83 & 75 & 47 & 61 & 28 & 59 & 22 \\
\hline J9 & 71 & 49 & 36 & 87 & 8 & 25 & 76 & 73 & 80 & 6 & 6 & 33 & 79 & 10 & 93 & 65 & 26 & 73 & 42 & 18 \\
\hline J10 & 7 & 40 & 33 & 64 & 5 & 25 & 89 & 95 & 58 & 83 & 28 & 35 & 74 & 5 & 6 & 9 & 3 & 2 & 35 & 41 \\
\hline J11 & 49 & 49 & 15 & 18 & 65 & 55 & 1 & 79 & 10 & 37 & 77 & 80 & 79 & 84 & 93 & 21 & 85 & 64 & 46 & 35 \\
\hline J12 & 3 & 53 & 59 & 7 & 65 & 58 & 24 & 55 & 26 & 40 & 89 & 94 & 51 & 74 & 54 & 86 & 22 & 83 & 19 & 44 \\
\hline J13 & 60 & 88 & 15 & 26 & 11 & 16 & 55 & 59 & 81 & 53 & 92 & 23 & 55 & 79 & 13 & 89 & 2 & 17 & 97 & 41 \\
\hline J14 & 12 & 47 & 46 & 17 & 43 & 16 & 91 & 94 & 73 & 89 & 12 & 58 & 25 & 24 & 55 & 1 & 67 & 3 & 1 & 71 \\
\hline J15 & 75 & 19 & 60 & 87 & 27 & 48 & 72 & 88 & 48 & 59 & 74 & 86 & 49 & 94 & 15 & 95 & 41 & 94 & 15 & 71 \\
\hline
\end{tabular}




\begin{tabular}{|c|c|c|c|c|c|c|c|c|c|c|c|c|c|c|c|c|c|c|c|c|}
\hline \multicolumn{11}{|c|}{ Case 9 (20x20): Taillard (1993) } \\
\hline $\mathrm{J} 16$ & 31 & 61 & 47 & 32 & 34 & 69 & 32 & 1 & 1 & 80 & 19 & 57 & 98 & 37 & 31 & 51 & 66 & 38 & 62 & 72 \\
\hline $\mathrm{J} 17$ & 70 & 78 & 41 & 9 & 47 & 94 & 26 & 65 & 17 & 42 & 59 & 80 & 7 & 75 & 63 & 96 & 7 & 10 & 47 & 38 \\
\hline $\mathrm{J} 18$ & 20 & 78 & 38 & 26 & 64 & 62 & 11 & 38 & 68 & 37 & 74 & 9 & 65 & 16 & 38 & 85 & 50 & 62 & 39 & 97 \\
\hline $\mathrm{J} 19$ & 88 & 30 & 34 & 33 & 21 & 7 & 94 & 10 & 73 & 85 & 82 & 62 & 99 & 67 & 61 & 10 & 4 & 70 & 31 & 49 \\
\hline $\mathrm{J} 20$ & 9 & 41 & 22 & 34 & 83 & 55 & 3 & 8 & 75 & 30 & 57 & 65 & 89 & 60 & 90 & 84 & 74 & 17 & 2 & 19 \\
\hline
\end{tabular}

(i)

\begin{tabular}{|c|c|c|c|c|c|c|c|c|c|c|}
\hline \multicolumn{11}{|c|}{ Case 10 (50x10): Taillard (1993) } \\
\hline$J \backslash M$ & M1 & $\mathrm{M} 2$ & M3 & M4 & M5 & M6 & M7 & M8 & M9 & M10 \\
\hline $\mathrm{J} 1$ & 46 & 61 & 3 & 51 & 37 & 79 & 83 & 22 & 27 & 24 \\
\hline $\mathrm{J} 2$ & 52 & 87 & 1 & 24 & 16 & 93 & 87 & 29 & 92 & 47 \\
\hline $\mathrm{J3}$ & 79 & 51 & 58 & 21 & 42 & 68 & 38 & 99 & 75 & 39 \\
\hline $\mathrm{J4}$ & 45 & 25 & 85 & 57 & 47 & 75 & 38 & 25 & 94 & 66 \\
\hline J5 & 97 & 73 & 33 & 69 & 94 & 37 & 86 & 98 & 18 & 41 \\
\hline J6 & 10 & 93 & 71 & 51 & 14 & 44 & 67 & 55 & 41 & 46 \\
\hline $\mathrm{J7}$ & 44 & 28 & 58 & 50 & 94 & 34 & 23 & 80 & 37 & 24 \\
\hline $\mathrm{J} 8$ & 24 & 90 & 56 & 51 & 34 & 39 & 19 & 82 & 58 & 23 \\
\hline J9 & 85 & 94 & 64 & 21 & 72 & 76 & 97 & 33 & 56 & 68 \\
\hline $\mathrm{J} 10$ & 75 & 59 & 43 & 19 & 36 & 62 & 78 & 68 & 20 & 50 \\
\hline $\mathrm{J} 11$ & 66 & 64 & 48 & 63 & 88 & 74 & 66 & 47 & 2 & 93 \\
\hline $\mathrm{J} 12$ & 49 & 2 & 69 & 91 & 51 & 28 & 67 & 74 & 39 & 22 \\
\hline $\mathrm{J} 13$ & 95 & 16 & 96 & 11 & 41 & 78 & 7 & 26 & 91 & 64 \\
\hline $\mathrm{J} 14$ & 61 & 35 & 35 & 6 & 71 & 43 & 23 & 61 & 81 & 81 \\
\hline $\mathrm{J} 15$ & 19 & 53 & 82 & 31 & 94 & 98 & 67 & 95 & 33 & 94 \\
\hline $\mathrm{J} 16$ & 47 & 40 & 53 & 63 & 99 & 83 & 8 & 55 & 14 & 97 \\
\hline $\mathrm{J} 17$ & 84 & 81 & 64 & 36 & 11 & 91 & 77 & 11 & 88 & 54 \\
\hline $\mathrm{J} 18$ & 13 & 26 & 11 & 39 & 97 & 27 & 71 & 42 & 22 & 82 \\
\hline $\mathrm{J} 19$ & 11 & 85 & 61 & 57 & 44 & 6 & 85 & 72 & 36 & 11 \\
\hline $\mathrm{J} 20$ & 19 & 4 & 36 & 47 & 77 & 82 & 29 & 14 & 65 & 91 \\
\hline $\mathrm{J} 21$ & 98 & 4 & 53 & 56 & 69 & 60 & 49 & 8 & 79 & 23 \\
\hline $\mathrm{J} 22$ & 2 & 10 & 87 & 65 & 91 & 44 & 3 & 98 & 23 & 32 \\
\hline $\mathrm{J} 23$ & 85 & 63 & 88 & 59 & 38 & 43 & 94 & 90 & 66 & 26 \\
\hline $\mathrm{J} 24$ & 44 & 96 & 10 & 4 & 25 & 76 & 76 & 36 & 5 & 22 \\
\hline $\mathrm{J} 25$ & 7 & 55 & 32 & 10 & 87 & 99 & 95 & 75 & 15 & 12 \\
\hline $\mathrm{J} 26$ & 73 & 71 & 38 & 12 & 7 & 66 & 48 & 69 & 51 & 23 \\
\hline $\mathrm{J} 27$ & 19 & 66 & 25 & 62 & 66 & 11 & 4 & 26 & 2 & 34 \\
\hline $\mathrm{J} 28$ & 69 & 94 & 24 & 43 & 54 & 35 & 37 & 24 & 81 & 87 \\
\hline $\mathrm{J} 29$ & 12 & 7 & 90 & 49 & 86 & 52 & 82 & 55 & 12 & 59 \\
\hline $\mathrm{J} 30$ & 73 & 15 & 7 & 54 & 49 & 8 & 57 & 98 & 40 & 2 \\
\hline $\mathrm{J} 31$ & 85 & 11 & 11 & 87 & 3 & 40 & 61 & 86 & 59 & 38 \\
\hline $\mathrm{J} 32$ & 23 & 99 & 49 & 29 & 48 & 62 & 6 & 30 & 32 & 84 \\
\hline $\mathrm{J} 33$ & 53 & 37 & 2 & 2 & 44 & 25 & 97 & 92 & 16 & 62 \\
\hline $\mathrm{J} 34$ & 16 & 50 & 76 & 18 & 93 & 24 & 5 & 94 & 87 & 10 \\
\hline $\mathrm{J} 35$ & 88 & 56 & 17 & 75 & 37 & 30 & 27 & 66 & 78 & 11 \\
\hline $\mathrm{J} 36$ & 8 & 69 & 32 & 39 & 82 & 1 & 95 & 47 & 41 & 93 \\
\hline $\mathrm{J} 37$ & 26 & 22 & 39 & 77 & 31 & 73 & 46 & 3 & 43 & 57 \\
\hline $\mathrm{J} 38$ & 42 & 56 & 9 & 69 & 59 & 27 & 92 & 41 & 94 & 81 \\
\hline $\mathrm{J} 39$ & 58 & 67 & 83 & 15 & 78 & 16 & 46 & 41 & 1 & 10 \\
\hline $\mathrm{J} 40$ & 63 & 63 & 69 & 78 & 33 & 91 & 52 & 47 & 93 & 40 \\
\hline $\mathrm{J} 41$ & 7 & 96 & 67 & 68 & 36 & 33 & 8 & 89 & 22 & 62 \\
\hline $\mathrm{J} 42$ & 2 & 74 & 28 & 37 & 3 & 11 & 11 & 28 & 93 & 49 \\
\hline $\mathrm{J} 43$ & 44 & 4 & 88 & 22 & 58 & 99 & 7 & 39 & 62 & 90 \\
\hline $\mathrm{J} 44$ & 38 & 42 & 23 & 41 & 10 & 2 & 54 & 80 & 53 & 34 \\
\hline $\mathrm{J} 45$ & 24 & 40 & 91 & 92 & 98 & 60 & 72 & 47 & 30 & 11 \\
\hline
\end{tabular}




\begin{tabular}{|c|c|c|c|c|c|c|c|c|c|c|}
\hline \multicolumn{10}{|c|}{ Case 10 (50x10): Taillard (1993) } \\
\hline $\mathrm{J} 46$ & 76 & 30 & 71 & 67 & 6 & 90 & 57 & 57 & 34 & 81 \\
\hline $\mathrm{J} 47$ & 85 & 93 & 3 & 24 & 44 & 36 & 85 & 74 & 27 & 51 \\
\hline $\mathrm{J} 48$ & 61 & 36 & 26 & 87 & 62 & 62 & 22 & 38 & 30 & 21 \\
\hline $\mathrm{J} 49$ & 32 & 25 & 41 & 91 & 24 & 15 & 87 & 59 & 54 & 39 \\
\hline $\mathrm{J} 50$ & 90 & 87 & 96 & 31 & 94 & 3 & 65 & 5 & 77 & 27 \\
\hline
\end{tabular}

(j)

Fig. 3 Input data set for testing the efficiency of the proposed heuristic technique $(a-j)$

\begin{tabular}{|c|c|c|c|c|c|c|c|}
\hline $\begin{array}{c}\text { Case (\#) } \\
\mathrm{J} \times \mathrm{M}\end{array}$ & Method & Sequence & Makespan & \begin{tabular}{|c|} 
Case (\#) \\
$\mathrm{J} \times \mathrm{M}$
\end{tabular} & Method & Sequence & Makespan \\
\hline \multirow{7}{*}{$\begin{array}{l}(1) \\
4 \times 4\end{array}$} & Palmer Slop Index & $3-1-2-4$ & 30 & \multirow{7}{*}{$\begin{array}{l}(5) \\
8 \times 3\end{array}$} & Palmer Slop Index & $8-2-6-4-7-3-1-5$ & 52 \\
\hline & Gupta's Heuristics & $3-1-2-4$ & 30 & & Gupta's Heuristics & $6-8-2-7-5-4-3-1$ & 55 \\
\hline & CDS & $3-1-2-4$ & 30 & & CDS & $8-2-6-3-7-4-5-1$ & 51 \\
\hline & Dannenbring & $3-2-1-4$ & 31 & & Dannenbring & $6-8-2-7-5-3-4-1$ & 52 \\
\hline & Hundal & $3-1-2-4$ & 30 & & Hundal & $8-2-7-6-3-4-5-1$ & 51 \\
\hline & Harmonic Triangle & $3-2-1-4$ & 31 & & Harmonic Triangle & $8-2-7-5-4-1-6-3$ & 59 \\
\hline & This Paper & 3-1-2-4 & 30 & & This Paper & $8-2-6-7-4-3-5-1$ & 51 \\
\hline \multirow{7}{*}{$\begin{array}{l}(2) \\
5 \times 5\end{array}$} & Palmer Slop Index & $4-2-1-5-3$ & 90 & \multirow{7}{*}{$\begin{array}{c}(6) \\
10 \times 8\end{array}$} & Palmer Slop Index & $9-3-4-6-10-8-7-5-1-2$ & 92 \\
\hline & Gupta's Heuristics & $4-1-2-3-5$ & 89 & & Gupta's Heuristics & $4-6-9-7-10-8-3-5-2-1$ & 96 \\
\hline & CDS & $4-1-2-3-5$ & 89 & & CDS & $9-4-6-10-3-7-5-1-8-2$ & 93 \\
\hline & Dannenbring & $3-1-2-5-4$ & 93 & & Dannenbring & $6-9-4-10-8-3-7-5-2-1$ & 92 \\
\hline & Hundal & $1-2-3-4-5$ & 89 & & Hundal & $9-4-3-6-10-8-7-1-5-2$ & 90 \\
\hline & Harmonic Triangle & $3-1-5-4-2$ & 90 & & Harmonic Triangle & $9-6-8-10-2-4-7-5-3-1$ & 94 \\
\hline & This Paper & $3-4-1-2-5$ & 89 & & This Paper & $9-4-3-6-10-8-7-5-1-2$ & 92 \\
\hline \multirow{7}{*}{$\begin{array}{l}(3) \\
5 \times 6\end{array}$} & Palmer Slop Index & $5-4-2-3-1$ & 225 & \multirow{7}{*}{$\begin{array}{c}(7) \\
10 \times 10\end{array}$} & Palmer Slop Index & $3-1-10-5-6-2-8-7-9-4$ & 99 \\
\hline & Gupta's Heuristics & $1-2-3-4-5$ & 229 & & Gupta's Heuristics & $3-6-10-1-5-9-8-2-7-4$ & 103 \\
\hline & CDS & $2-3-4-5-1$ & 224 & & CDS & $3-6-10-1-9-5-8-2-7-4$ & 102 \\
\hline & Dannenbring & $1-2-3-4-5$ & 229 & & Dannenbring & $3-10-1-5-9-8-6-2-7-4$ & 97 \\
\hline & Hundal & $2-1-5-3-4$ & 214 & & Hundal & $3-1-10-6-5-2-8-7-4-9$ & 100 \\
\hline & Harmonic Triangle & $1-2-3-4-5$ & 229 & & Harmonic Triangle & $3-6-1-10-9-5-4-2-7-8$ & 96 \\
\hline & This Paper & $2-1-5-3-4$ & 214 & & This Paper & $3-1-10-5-6-8-2-9-7-4$ & 97 \\
\hline \multirow{7}{*}{$\begin{array}{l}(4) \\
7 \times 7\end{array}$} & Palmer Slop Index & $4-1-5-3-2-7-6$ & 70 & \multirow{7}{*}{$\begin{array}{c}(8) \\
20 \times 10\end{array}$} & Palmer Slop Index & $5-12-15-17-18-4-9-2-20-3-10-13-11-19-6-7-14-8-16-1$ & 1790 \\
\hline & Gupta's Heuristics & $4-1-3-5-2-7-6$ & 70 & & Gupta's Heuristics & 2-8-17-12-5-9-6-4-15-19-13-10-7-11-3-18-20-14-16-1 & 2027 \\
\hline & CDS & $4-1-3-5-6-2-7$ & 68 & & CDS & $2-17-18-5-9-15-12-20-4-6-7-13-19-10-14-8-11-3-1-16$ & 1757 \\
\hline & Dannenbring & $4-1-3-5-6-2-7$ & 68 & & Dannenbring & $18-2-17-5-3-4-9-12-15-20-10-13-8-19-14-11-7-6-1-11$ & 1771 \\
\hline & Hundal & $4-5-3-1-2-7-6$ & 69 & & Hundal & $5-12-18-17-15-4-2-9-3-20-10-11-6-13-7-19-14-8-16-1$ & 1741 \\
\hline & Harmonic Triangle & $1-7-3-5-6-2-4$ & 78 & & Harmonic Triangle & $16-2-6-4-17-5-9-8-13-20-14-10-19-12-11-15-7-1-3-18$ & 2016 \\
\hline & This Paper & 4-1-5-3-2-6-7 & 66 & & This Paper & 5-12-18-15-17-4-9-2-3-20-10-13-19-11-7-6-14-8-1-16 & 1758 \\
\hline \multirow{7}{*}{$\begin{array}{c}(9) \\
20 \times 20\end{array}$} & Palmer Slop Index & \multicolumn{5}{|c|}{$11-16-12-18-3-7-20-5-13-15-1-6-19-9-14-8-17-10-2-4$} & 2466 \\
\hline & Gupta's Heuristics & \multirow{2}{*}{\multicolumn{5}{|c|}{ 16-14-10-20-3-7-5-1-18-12-15-6-8-4-11-13-17-19-2-9 }} & 2555 \\
\hline & CDS & \multirow{2}{*}{\multicolumn{5}{|c|}{$\frac{20-3-12-14-11-18-7-16-2-6-5-15-1-8-9-13-19-4-17-10}{16-20-18-12-11-18-5-3-19-7-6-1-15-8-2-9-17-14-4-10}$}} & 2493 \\
\hline & Dannenbring & & & & & & 2568 \\
\hline & Hundal & \multicolumn{5}{|c|}{$11-12-16-18-7-3-5-20-15-1-6-13-19-9-14-8-17-2-10-4$} & 2536 \\
\hline & Harmonic Triangle & \multicolumn{5}{|c|}{$20-14-12-5-10-3-18-7-16-1-6-13-17-4-15-11-19-9-2-8$} & 2580 \\
\hline & This Paper & \multicolumn{5}{|c|}{$11-16-18-12-3-20-7-5-13-1-15-6-19-9-14-8-17-2-10-4$} & 2492 \\
\hline \multirow{7}{*}{$\begin{array}{c}(10) \\
50 \times 10\end{array}$} & Palmer Slop Index & \multicolumn{5}{|c|}{$\begin{array}{c}\text { 20-38-18-36-15-14-33-43-42-2-44-29-4-49-31-25-37-34-22-16-3-46-28-30-6-12-32-13-41-40-7-10-8-11-1-47-19-26-17-9-23-21- } \\
24-35-45-48-27-5-39-50 \\
\end{array}$} & 3461 \\
\hline & Gupta's Heuristics & \multicolumn{5}{|c|}{$\begin{array}{c}33-22-42-27-29-20-25-32-18-49-14-41-43-37-11-6-28-16-4-38-36-15-46-40-9-23-50-3-5-8-7-21-35-10-48-12-1-17-19-45-13-34- \\
47-2-31-30-26-24-44-39\end{array}$} & 3672 \\
\hline & CDS & \multicolumn{5}{|c|}{$\begin{array}{c}\text { 18-20-37-33-25-30-49-22-44-42-31-38-29-36-12-7-14-43-2-16-34-15-4-41-28-3-23-13-40-46-8-9-5-35-17-47-32-26-11-6-10-19- } \\
21-50-48-45-1-24-27-39\end{array}$} & 3421 \\
\hline & Dannenbring & \multicolumn{5}{|c|}{$\begin{array}{c}\text { 42-44-18-33-20-37-49-14-22-36-34-31-43-25-29-2-38-4-28-16-3-46-15-9-40-23-5-11-17-45-13-47-10-6-12-41-50-21-7-8-32-19- } \\
35-26-1-30-48-24-39-27\end{array}$} & 3510 \\
\hline & Hundal & \multicolumn{5}{|c|}{$\begin{array}{c}20-18-38-36-33-14-42-43-15-44-2-49-29-4-37-31-25-22-34-30-16-3-46-28-32-6-12-41-13-7-1-8-10-19-26-40-47-11-24-27-17-21- \\
35-48-9-23-45-5-39-50\end{array}$} & 3469 \\
\hline & Harmonic Triangle & \multicolumn{5}{|c|}{$\begin{array}{c}27-29-22-25-18-41-36-24-19-45-32-6-37-39-16-15-1-11-33-20-8-42-3-10-46-47-38-9-28-17-2-50-14-40-5-4-43-23-26-13-44-34- \\
35-49-7-48-31-21-12-30\end{array}$} & 3856 \\
\hline & This Paper & \multicolumn{5}{|c|}{$\begin{array}{c}\text { 20-18-33-42-36-38-14-43-15-44-2-49-29-4-31-25-37-22-34-16-3-46-28-6-30-12-13-32-41-40-7-11-10-8-47-1-17-9-19-26-23-21-5 } \\
45-35-24-48-27-50-39\end{array}$} & 3415 \\
\hline
\end{tabular}

Fig. 4 Comparative results between the proposed technique and six benchmark techniques

It is clear from the table presented in Figure 4 that the proposed technique is more effective than the other heuristic techniques in all of the ten tested flow shop problems. Figure 4 shows the makespan for each of the ten problems for all six methods including the method proposed in this study. Table 3 shows that the proposed technique gives a better result than the harmonic triangle, Dannenbring, Palmer, Gupta, CDS, and Hundal methods by $90 \%, 80 \%$, $70 \%, 70 \%, 60 \%$, and $40 \%$, respectively. 
Table 3 A comparison between the proposed method and six other methods

\begin{tabular}{lllll}
\hline Other methods & $\begin{array}{l}\text { Number of } \\
\text { times when the } \\
\text { solution by the } \\
\text { proposed } \\
\text { method is } \\
\text { better }\end{array}$ & $\begin{array}{l}\text { Number of } \\
\text { times when the } \\
\text { solution by the } \\
\text { proposed } \\
\text { method is } \\
\text { equal }\end{array}$ & $\begin{array}{l}\text { Number of } \\
\text { times when the } \\
\text { solution } \\
\text { achieved by } \\
\text { other methods } \\
\text { is better }\end{array}$ & $\begin{array}{l}\text { Percentage of } \\
\text { proposed } \\
\text { method when it } \\
\text { is better than } \\
\text { other method }\end{array}$ \\
\hline Palmer Slope Index & 7 & 2 & 1 & $70.00 \%$ \\
Gupta's Heuristics & 7 & 3 & --- & $70.00 \%$ \\
CDS & 6 & 3 & 1 & $60.00 \%$ \\
Dannenbring & 8 & 2 & -- & $80.00 \%$ \\
Hundal & 4 & 4 & 2 & $40.00 \%$ \\
Harmonic Triangle & 8 & --- & 1 & $88.89 \%$ \\
\hline
\end{tabular}

As shown in Table 3, the proposed method has a better result in seven out of ten problems compared to the Palmer Slope Index, in seven problems compared to Gupta's, in six problems compared to CDS, in eight problems compared to Dannenbring, in four problems compared to Hundal, and in eight problems compared to the harmonic triangle. Table 4 shows the percentage of improvement provided by the proposed method corresponding to the problem size with respect to each of the other methods. Moreover, Table 4 indicates that the average improvement of the proposed technique over the other six methods is notable. The proposed technique has improved the results on average by $6.86 \%, 4.81 \%, 2.53 \%, 1.77 \%$, $1.35 \%$, and $0.74 \%$ over the harmonic triangle, Gupta, Dannenbring, Palmer, CDS, and Hundal, respectively.

Table 4 Percentage of improvement achieved by proposed method compared to other methods

\begin{tabular}{|c|c|c|c|c|c|c|}
\hline $\begin{array}{l}\text { Problem size } \\
\qquad(\mathrm{N} \times \mathrm{M})\end{array}$ & $\begin{array}{l}\text { Palmer } \\
\text { Slope } \\
\text { Index }\end{array}$ & $\begin{array}{l}\text { Gupta's } \\
\text { Heuristics }\end{array}$ & CDS & Dannenbring & Hundal & $\begin{array}{c}\text { Harmonic } \\
\text { Triangle }\end{array}$ \\
\hline $4 \times 4$ & $0.00 \%$ & $0.00 \%$ & $0.00 \%$ & $3.23 \%$ & $0.00 \%$ & $3.23 \%$ \\
\hline $5 \times 5$ & $1.11 \%$ & $0.00 \%$ & $0.00 \%$ & $4.30 \%$ & $0.00 \%$ & $1.11 \%$ \\
\hline $5 \times 6$ & $4.89 \%$ & $6.55 \%$ & $4.46 \%$ & $6.55 \%$ & $0.00 \%$ & $6.55 \%$ \\
\hline $7 \times 7$ & $5.71 \%$ & $5.71 \%$ & $2.94 \%$ & $2.94 \%$ & $4.35 \%$ & $15.38 \%$ \\
\hline $8 \times 3$ & $1.92 \%$ & $7.27 \%$ & $0.00 \%$ & $1.92 \%$ & $0.00 \%$ & $13.56 \%$ \\
\hline $10 \times 8$ & $0.00 \%$ & $0.00 \%$ & $1.08 \%$ & $0.00 \%$ & $-2.22 \%$ & $2.13 \%$ \\
\hline $10 \times 10$ & $2.02 \%$ & $5.83 \%$ & $4.90 \%$ & $0.00 \%$ & $3.00 \%$ & $-1.04 \%$ \\
\hline $20 \times 10$ & $1.79 \%$ & $13.27 \%$ & $-0.06 \%$ & $0.73 \%$ & $-0.98 \%$ & $12.80 \%$ \\
\hline $20 \times 20$ & $-1.05 \%$ & $2.47 \%$ & $0.04 \%$ & $2.96 \%$ & $1.74 \%$ & $3.41 \%$ \\
\hline $50 \times 10$ & $1.35 \%$ & $7.00 \%$ & $0.18 \%$ & $2.71 \%$ & $1.56 \%$ & $11.44 \%$ \\
\hline $\begin{array}{c}\text { Average } \\
\text { improvement }\end{array}$ & $1.77 \%$ & $4.81 \%$ & $1.35 \%$ & $2.53 \%$ & $0.74 \%$ & $6.86 \%$ \\
\hline
\end{tabular}




\section{Conclusion}

The primary purpose of the paper was to develop and demonstrate a new heuristic technique to solve ' $n$ ' jobs on ' $m$ ' machines for static flow shop sequencing problems with a minimum makespan time as an objective function. The proposed technique assigns a slope index to each job based on its trend journey from the first to the last machine. After that, the indices are ranked in descending order to determine the job sequencing and corresponding makespan time. The technique is simple and quick to produce one sequence at a time. Based on ten tested flow shop problems with sizes ranging from $4 \times 4$ to $50 \times 10$, a comparative analysis is made to find the effectiveness of the proposed technique with respect to the other five well-known heuristics (Palmer, Gupta, CDS, Dannenbring, and Hundal) as well as a recent one (harmonic triangle). The results showed that the proposed technique successfully improved (on average) the makespan time over the other six heuristics. As an overall observation, the proposed technique is more efficient than the harmonic triangle methodology, while the Hundal technique is the closest competitor to the proposed technique. In future work, examining the proposed technique on a job shop, or extending the proposed technique to a dynamic job shop might be an area of study.

\section{REFERENCES}

[1] Y. MarfoMissah, "Business Innovation with Enterprise Architecture," Int. J. Comput. Appl., vol. 120, no. 9, pp. 12-15, 2015, https://doi.org/10.5120/21254-4097

[2] S. M. Johnson, "Optimal two- and three-stage production schedules with setup times included," Nav. Res. Logist. Q., vol. 1, no. 1, pp. 61-68, Mar. 1954, https://doi.org/10.1002/NAV.3800010110

[3] D. Palmer, "Sequencing Jobs Through a Multi-Stage Process in the Minimum Total Time - A Quick Method of Obtaining a Near Optimum," J. Oper. Res. Soc., vol. 16, pp. 101-107, 1965, https://doi.org/10.1057/jors.1965.8

[4] T. Meng and Q.-K. Pan, “A distributed heterogeneous permutation flowshop scheduling problem with lot-streaming and carryover sequence-dependent setup time," Swarm Evol. Comput., vol. 60, 2021, https://doi.org/10.1016/j.swevo.2020.100804

[5] H. G. Campbell, R. A. Dudek, and M. L. Smith, “A A Heuristic Algorithm for the n Job, m Machine Sequencing Problem ," Manage. Sci., vol. 16, no. 10, p. B-630-B-637, Jun. 1970, https://doi.org/10.1287/MNSC.16.10.B630

[6] J. N. D. Gupta, "A heuristic algorithm for the flowshop scheduling problem," RAIRO - Oper. Res. - Rech. Opérationnelle, vol. 10, no. V2, pp. 63-73, 1976, Accessed: Aug. 03, 2021. [Online]. Available: http://www.numdam.org/item?id=RO_1976_10_2_63_0. https://doi.org/10.1051/ro/197610V200631

[7] I. Ribas, R. Companys, and X. Tort-Martorell, "Efficient heuristics for the parallel blocking flow shop scheduling problem," Expert Syst. Appl., vol. 74, pp. 41-54, 2017, https://doi.org/10.1016/j.eswa.2017.01.006

[8] D. G. Dannenbring, “An Evaluation of Flow Shop Sequencing Heuristics,” Manage. Sci., vol. 23, no. 11, 1977, https://doi.org/10.1287/mnsc.23.11.1174

[9] A. Garay-Sianca and S. G. Nurre Pinkley, "Interdependent integrate d network design and sche duling problems with movement of machines," European Journal of Operational Research, Volume 289, Issue 1, pp. 297-327, 2021, https://doi.org/10.1016/j.ejor.2020.07.013

[10] T. S. HUNDAL and J. RAJGOPAL, “An extension of Palmer's heuristic for the flow shop scheduling problem," International Journal of Production Research, vol. 26, no. 6, pp. 1119-1124, 1988, https://doi.org/10.1080/00207548808947922

[11] B. Dhanasakkaravarthi and A. Krishnamoorthy, "A NEW HEURISTIC ALGORITHM TO DETERMINE MORE THAN ONE SEQUENCE IN PERMUTATION FLOW SHOP SCHEDULING BY USING HARMONIC TRIANGLE,” Int. J. Mech. Eng. Technol., vol. 10, no. 03, pp. 284-289, 2019.

[12] A. K. Agarwal and R. Garg, “Advanced Modelistic Approach of Flowshop Scheduling Problem for 10Jobs , 10-Machines By Heuristics Models Using Makespan Criterion,” Int. J. Eng. trend Technol., vol. 4, no. 5, pp. 1742-1748, 2013. 
[13] E. Taillard, "Benchmarks for basic scheduling problems," Eur. J. Oper. Res., vol. 64, no. 2, pp. 278-285, 1993, https://doi.org/10.1016/0377-2217(93)90182-M

[14] E. Vallade, R. Ruiz, and J. M. Framinan, "New hard benchmark for flowshop scheduling problems minimising makespan,” Eur. J. Oper. Res., vol. 240, no. 3, pp. 666-677, 2015, https://doi.org/10.1016/j.ejor.2014.07.033

Submitted: $\quad 27.08 .2021$

Accepted: $\quad 22.10 .2021$
Prof. Reda M. S. Abdulaal

The University Vice Presidency for Graduate Studies and Scientific Research, King Abdulaziz University, P.O. Box 80204 Jeddah 21589, Saudi Arabia Contact Number : +201001771091 ORCID : 0000-0001-8806-2115

Assist. Prof. Omer A. Bafail* Department of Industrial Engineering, College of Engineering, King Abdulaziz University, P.O. Box 80204 Jeddah 21589, Saudi Arabia

Contact Number: +966504674267

ORCID: 0000-0002-2586-7028

*Corresponding author: oabafail@kau.edu.sa 